\title{
Coherent Radiation from Free-Electron Lasers
}

The term "coherent radiation" is nowadays virtually automatically associated with the word laser. It is well known that the acronym laser is the briefest possible description of the mechanism by which atoms or molecules pumped into an excited state and having reached population inversion, are subsequently stimulated to decay coherently. In this strict definition, the free-electron laser (FEL) discussed here is not a laser. However, one may relax the definition by ignoring the nature of the lasing medium and consider a laser to be any device that initially produces weak and incoherent spontaneous emission, but which can be made to emit powerful, coherent radiation by capturing the spontaneous radiation in an optical cavity. Following the latter sense, the FEL is a special member of the laser family.

In a FEL, radiation is produced as a result of coherent oscillations of free electrons instead of electrons bound in atoms. The principle of operation is sketched in Fig. 1: a beam of relativistic electrons is injected into a periodic magnetic structure (a so-called undulator, or a wiggler with a similar construction but a much longer magnetic period). The spatially alternating magnetic field causes the electrons to oscillate, resulting in the emission of radiation, essentially incoherent, that is captured in an optical resonator. The stored field induces newly injected electrons to radiate coherently, resulting in the build-up of a high laser power. At saturation, the stored power is typically eight to ten orders of magnitude more intense than the initial radiation. As with other lasers, a fraction of the stored power is coupled out through one of the cavity mirrors.

The FEL wavelength is determined by macroscopic parameters (namely the electron energy and the period and strength of the undulator field) rather than by the microscopic properties of atoms. This striking distinction from other types of lasers means that FELs are designed to operate at any wavelength, while the wavelength can at the same time be scanned continuously by varying one of the macroscopic parameters. Another difference is that there is no danger

P.W. van Amersfoort studied physics at Delft University of Technology and in 1982 joined the FOM-Institute for Plasma Physics, Postbus 1207, NL-3430 BE Nieuwegein. In 1987, he was awarded a Ph.D. for work on negative ion formation and became the leader of the Institute's FELIX free-electron laser project.

M.J. van der Wiel is the Director of the FOM-Institute for Plasma Physics. He studied at the University of Amsterdam, receiving his Ph.D. in 1971. After working as a postdoc in the Chemistry Department, University of British Colombia, Vancouver, he returned to Amsterdam, becoming in 1978 Professor of Physics at the Free University and Deputy-Director of the FOM-Institute for Atomic and Molecular Physics.

\section{P.W. van Amersfoort, M.J. van der Wiel}

FOM-Institute for Plasma Physics, Nieuwegein, The Netherlands

\section{FELIX Inauguration}

The FELIX free-electron laser facility will be officially inaugurated on 21 December by Holland's Secretary of State for Higher Education and Science Policy. It is also the occasion for the signing of an agreement between the UK's SERC, and The Netherlands' NWO and FOM funding organizations to cover access by UK scientists for an initial two-year period to $20 \%$ of FELIX's beam time (a review in the second year will consider an extension). With the agreement, FELIX will be officially recognized as an international facility for which additional funds are made available.

The FOM-Institute for Plasma Physics recently started construction of a free-electron maser (a mm-wavelength range laser) for producing microwaves to explore methods based on local heating to suppress plasma instabilities. Supported by EURATOM, the first radiation is planned for 1994 with full operation in 1996. Almost one-half of the project involves contributions from companies and other institutes.

of damaging the lasing medium, even at a high stored power, because the "medium" is the beam of electrons and it is effectively replenished every few nanoseconds. These properties make the FEL a versatile source of powerful, coherent radiation which is presently demonstrating its potential to open up applications in many new areas.

The history of the FEL is not without interest. As early as 1957, a device in the electron-tube family called an ubitron (an acronym for undulating beam interaction), but in essence an FEL "avant la lettre", was operated at General Electric in the USA, with R.M. Phillips as the principal investigator [1]. The only difference with the FEL was the much smaller electron energy (in fact only slightly relativistic) which implied that the output was in the microwave region. Although the ubitron broke records for radiofrequency power generation, research was stopped in 1964. The concept was re-introduced by J.M.J. Madey at Stanford University in 1971, and (with an excellent sense for public relations) advertised as a "free-electron laser". The new name emphasized that the amplification mechanism is distinctly different from that found in electron tubes such as klystrons and magnetrons. Madey and coworkers had measured by 1976 noticeable gain from a superconducting linac-based FEL configured as an amplifier at a wavelength of $10.6 \mu \mathrm{m}$. The same device (now configured as an oscillator) produced a saturated laser output at wavelength of $3.4 \mu \mathrm{m}$ about a year later.

These pioneering experiments led to worldwide interest in FEL research and in subsequent years, some $20 \mathrm{FEL}$ projects were undertaken at different laboratories with various degrees of success. The principles of operation are now well understood, and lasing has been achieved over a large spectral range, from the microwave region to the ultraviolet.

\section{Principles of Operation}

The generic layout of the FEL, as sketched in Fig. 1, comprises three components: an accelerator delivering an intense electron beam at relativistic energies, an undulator, and an optical cavity. Undulators, which are also frequently used in synchrotron radiation facilities, are usually made from permanent magnets. The emission of synchrotron radiation (known as spontaneous emission in the FEL community) is the starting point for a brief discussion of the FEL amplification process.

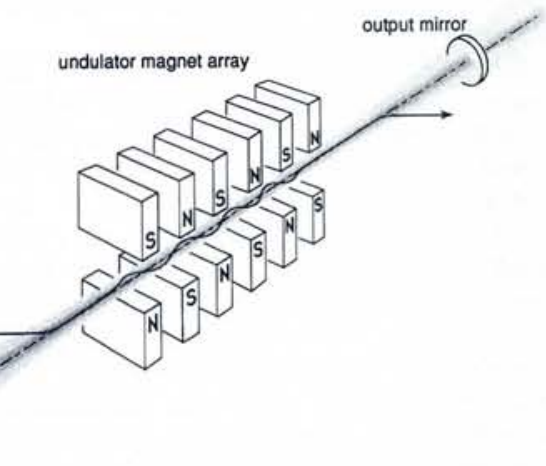

Fig. 1 - Principle of the free-electron laser (FEL). A beam of relativistic electrons is injected into a periodic magnetic structure, the so-called

undulator or wiggler. The magnetic field generates a periodically curved electron trajectory, and the oscillating dipole moment thus induced leads to the emission of radiation. This radiation is captured in an optical cavity and amplified on successive passes through the undulator in the presence of "fresh" electrons. The electrons are deflected out of the cavity at the exit to the undulator and a fraction of the stored power is removed through one of the cavity mirrors. 
In their rest frame, the electrons are at a fixed axial position and the static undulator field (with period $\lambda_{u}$ ) is transformed owing to a shifting of wavelength induced by relativistic effects (the Lorentz contraction) into a virtual electromagnetic wave with a reduced period $\lambda_{\mathrm{u}} / \gamma$ where $\gamma$ is the Lorentz factor. The electric component of the wave causes electrons to oscillate, and the oscillating dipole moment leads to the emission of radiation at the same wavelength as the undulator field. In the rest frame, the emission profile has the well-known dipole pattern, but when transformed back to the laboratory frame, the profile becomes strongly peaked in the forward direction. Moreover, the wavelength is Doppler-shifted by a factor $2 \gamma$ which leads to a wavelength a factor $2 \gamma^{2}$ smaller than the undulator period. For an electron energy of say $50 \mathrm{MeV}$, $\gamma=100$ with the result that the radiation wavelength is $2 \times 10^{4}$ times shorter than the undulator period. For a typical undulator period of $50 \mathrm{~mm}$, the wavelength is thus $2.5 \mu \mathrm{m}$, i.e., in the near-infrared.

The maximum spontaneous synchrotron radiation power is limited by the fact that the waves emitted by different electrons tend to interfere destructively. As illustrated in Fig. 2 , electrons at an axial separation of onequarter of a wavelength radiate $90^{\circ}$ out of phase so that the two waves are $180^{\circ}$ out of phase. The essence of FEL operation is that the initially homogeneous electron-line density is modulated in space to give peaks with a one-half wavelength separation. As shown in Fig. 2, dipole oscillators separated by this distance radiate in phase so that the fields add up coherently.

Density modulation is brought about by capturing the initial synchrotron radiation in an optical cavity. For newly injected electrons, this has the consequence that in addition to the virtual undulator wave travelling from right to left in Fig. 2, there is also a real electromagnetic wave travelling from the left to right. In the rest frame, the two waves have the same period resulting in a standing wave. The transverse velocity couples with the magnetic component to form an axial force, the so-called ponderomotive force, associated with the standing wave that drives the electrons away from the peaks of the electric field, as shown in Fig. 3. This causes electrons passing through the undulator to group into bunches with a separation of one-half the radiation wavelength.

Even though bunches are formed at the required axial separation, there is the problem that they are centred around positions where the stored electric field is in antiphase with the undulator field. In other words, the wave emitted by the oscillating electrons would be out of phase with the stored field, and the latter would not experience laser amplification. However, this problem is absent when there is a small mismatch between the undulator period and the radiation wavelength. In the rest frame, this has the consequence that all electrons have a small axial velocity, with a value depending on the degree of mismatch. The optimum axial velocity is such that, after initial bunching in the first part of the undulator, the bunches at the downstream end of the undulator

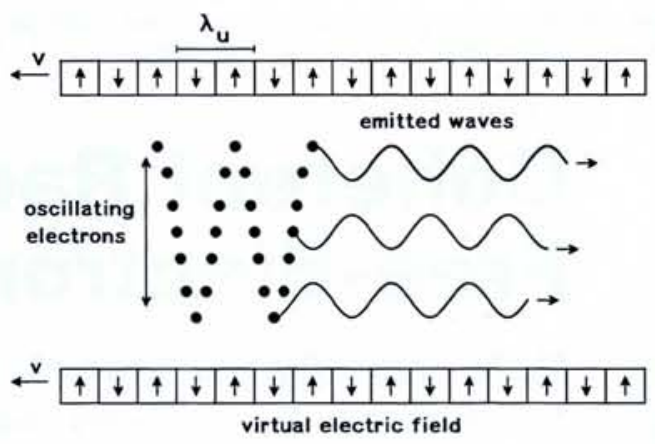

Fig. 2 - Emission of radiation by electrons moving through an undulator (of wavelength $\lambda_{u}$ ) at a velocity $v$. The situation in the rest frame is shown, where the electrons (represented as black dots) are at a fixed axial position and the undulator field is transformed into a virtual electromagnetic wave with velocity $v$. The electric field of the wave induces an oscillating dipole moment which leads to the emission of radiation. The waves emitted by dipoles at an axial separation of onequarter of a wavelength are $90^{\circ}$ out of phase, so they effectively cancel out. The essence of the FEL mechanism is that the electrons form bunches at an axial separation of one-half of a wavelength; waves emitted by different bunches then add up coherently.

\section{Fig. 3 - The standing wave} formed by the virtual undulator wave and the stored wave. The electric component leads to the transverse "wiggle" motion, and the transverse velocity couples with the magnetic component $B$ to form an axial force, the socalled ponderomotive force $F$. This force drives the electrons towards positions of minimum electric field strength $E$. This way, they group in bunches separated by one-half of a wavelength while travelling through the undulator.

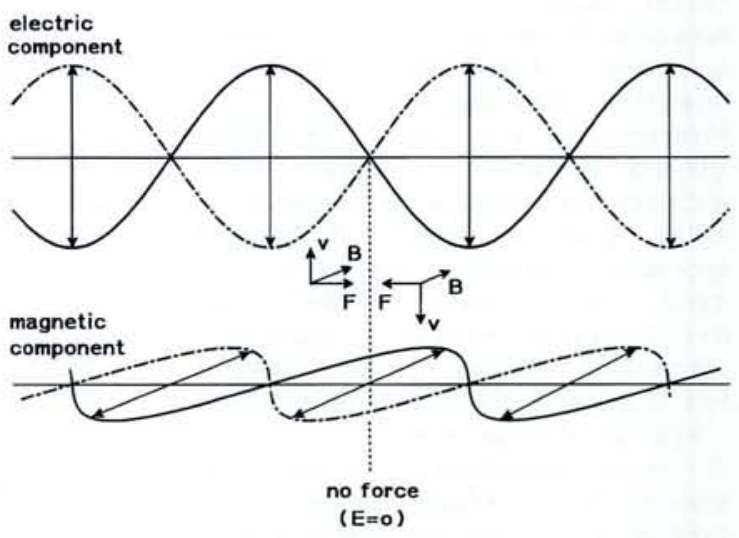

centre around peaks in the electric field so that the emitted radiation comes into phase with the stored field. The corresponding increase in stored power leads to more effective bunching of "fresh" (newly injected) electrons, thereby enhancing the emission of coherent radiation which in turn leads to more effective bunching of fresh electrons, and so on. At saturation, the stored power is typically eight to ten orders of magnitude higher than the initial synchrotron radiation power. No further increase in power occurs because the electrons lose so much energy that they become out of phase with the stored field.

\section{Operation in the Infrared}

Most existing FELs produce radiation in the infrared spectral range. An important reason for this is the relatively small electron energy (less than $50 \mathrm{MeV}$ ) that is required for operation in this part of the electromagnetic spectrum. Much effort has been expended in obtaining the excellent properties of electron beams needed for FEL oscillation (high brightness, small emittance, small energy spread, long pulse length, very good stability, etc.) This has pushed the state-of-the-art in electron accelerator technology and it is generally felt that sufficient experience with infrared FELs has been obtained to provide the reliability needed for application in user experiments. A number of FEL user facilities have been proposed worldwide since the mid-1980s: several are now operating and others are under construction (the spectral ranges covered by planned and existing facilities are summarized in Fig. 4.).

The oldest user facility, at Santa Barbara in the USA and operating in the far-infrared spectral range from $60 \mu \mathrm{m}$ to several $\mathrm{mm}$, came on-line in 1986. An electrostatic accelerator is used to produce a beam of electrons at a maximum energy of $6 \mathrm{MeV}$. In Europe, similar devices have been proposed recently by groups at Lille and Dijon. A proposed electrostatic accelerator-based FEL project at Oxford has not been funded although in a pilot experiment it produced radiation in the 350-1860 $\mu \mathrm{m}$ spectral range using a so-called Smith-Purcell configuration (i.e., a metal grating instead of a row of undulator magnets).

A beam energy of typically $10-50 \mathrm{MeV}$ is required for FELs operating in the mid-and near-infrared so a radiofrequency accelerating field is necessary. The most popular device here is the radiofrequency linac, an instrument used in numerous nuclear physics laboratories around the world. Two linac-based user facilities came into operation in 1992 in Europe, namely FELIX (see the cover illustration) at the FOM-Institute, Nieuwegein, which recieves regular support as an EURATOM Associate, and CLIO at LURE, Orsay. These FELs together cover the spectral range from 3 to $110 \mu \mathrm{m}$ which places them in a prominent position worldwide. A specific advantage of CLIO is the presence of the Super-ACO synchrotron radiation facility in the same building. Besides linacs, microtrons (a microtron is a type of relativistic cyclotron) are used as FEL injectors at the ENEA laboratory, Frascati, and at Twente University, the former having recently produced radiation in the microwave spectral range.

The electron beam produced by rf-linacs consists of a train of "micropulses" with a duration of a few ps. The optical beam therefore also comprises short, intense micropulses. The micropulse energy of FELIX is shown in Fig. 5 for wavelengths ranging 
from 6.5 to $110 \mu \mathrm{m}$. Rapid wavelength scans over an octave can be made by a straightforward adjustment of the undulator field strength, and FELIX is the first FEL where this kind of on-line tunability has been realised. In addition, the duration of the optical micropulses can be varied from 1 to 20 ps by adjusting the length of the cavity.

\section{Applications of Infrared FELs}

Conventional lasers with a high output power are only available for a limited number of specific wavelengths in the infrared spectral range (examples include the $\mathrm{CO}_{2}$ laser operating at $10.6 \mu \mathrm{m}$ and the Nd:YAG laser at $1.06 \mu \mathrm{m})$. In view of this, there exists a wealth of applications for infrared FELs providing high output powers over a large and continuous spectral range. Moreover, the short pulses of if accelerator-based FELs are well suited for studies of dynamic processes taking place on the time scale of a few nanoseconds, or less.

The applications of short-pulse infrared FELs can be broadly categorised as follows [2]: (i) electronic transitions of materials with small energy gaps; (ii) mechanical excitations; (iii) heating of nanoscale structures. The first category includes a variety of materials such as semiconductors, high- $T_{C}$ superconductors, and large organic and inorganic molecules with low-lying electronic states. The second includes vibrations localized on individual molecules, and phonons and vibrons, i.e. collective modes of crystalline solids. Vibrations are sensitive to the details of the structure of a molecule, and therefore provide fingerprint selectivity in molecular identification. Selective activation of a specific molecular vibration may provide routes for controlling the chemical reactivity of the molecule concerned. Vibrational spectroscopy can be used to investigate crystalline and amorphous solids, liquids, molecules on surfaces, and systems of biological or biomedical interest.

Regarding the third group of applications, the FEL differs from ordinary heat sources in its ability to excite one component of a complex mixture via vibrational selectivity to produce a substantial jump in temperature of extremely short duration. Examples of applications include the selective dissociation of molecules in a gas mixture, the selective desorption of molecules from a surface, photo-ablation of biological tissue, etc.

The unique properties of the FEL attract users from various scientific disciplines. Experiments for the FELIX user facility have been proposed by groups from atomic physics, molecular physics and chemistry, solid-state physics, surface physics and chemistry (including liquid-metal interfaces), and biomedicine. Of the 35 requests for beam time received so far, one-third are from solid-state physics. The mode of operation as a user facility differs to that for synchrotron sources where there are many beam lines. Radiation is delivered instead via a main beam line to one of six user stations for various lengths of time.

\section{Operation in the Visible and Ultraviolet}

In comparison with infrared FELs, less effort has been made to develop FELs for

Fig. 4-The spectral ranges covered by operational and planned FEL user facilities in Europe and the USA (the cross-hatched regions correspond to today's ranges and possible extensions are indicated by the boxes). Most facilities employ a radiofrequency field to accelerate the electrons, with the consequence that the laser output consists of a train of short, intense micropulses with a duration of a few ps. The most important exception is the FEL at Santa Barbara, USA, which uses an electrostatic accelerator. The

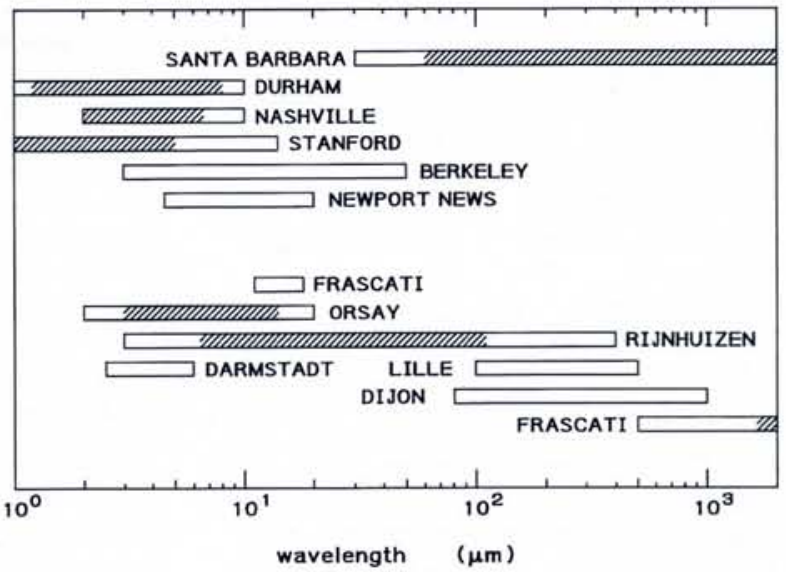

result is a quasi-continuous laser output, with a duration in the $\mu$ s range and a much smaller power. Similar FELs have been proposed by groups at Lille and Dijon.

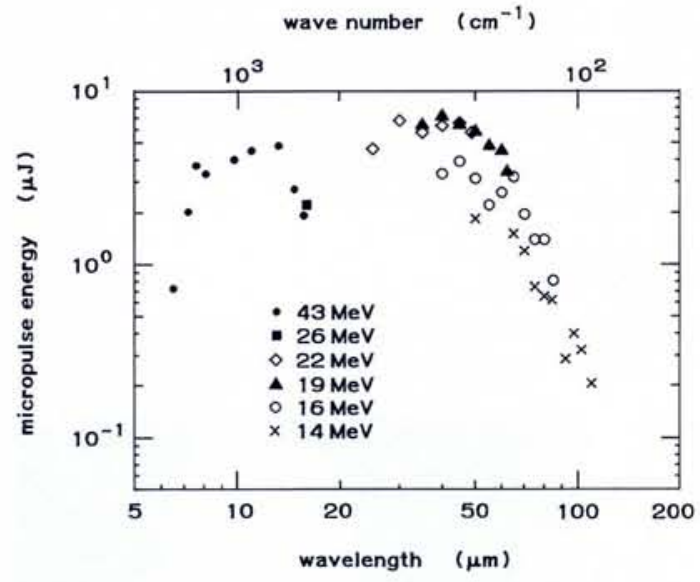

Fig. 5 - Energy of the optical micropulses of FELIX, for wavelengths ranging from 6.5 to 110 $\mu \mathrm{m}$, showing results for different electron beam energies. At a fixed electron energy, the wavelength can be scanned over an octave via adjustment of the undulator field. This simple procedure permits a full scan in typically two minutes. The duration of the optical micropulses can be varied by adjusting the length of the optical cavity (the present range is from 1 to 20 ps) and the corresponding peak power is $10 \mathrm{MW}$.

the visible or near-ultraviolet owing to the wide variety of bench-top, high-power laser systems available commercially. Research in this part of the spectrum is carried out mainly as a step towards the soft $x$-ray regime where conventional lasers are not available. This situation is related to a fundamental limitation: the spontaneous decay rate from upper to lower laser levels increases drastically with increasing photon energy as a consequence of the Einstein relations. Hence, on going to a very short wavelength it becomes increasingly difficult to create and maintain a population inversion.

The FEL does not suffer from this fundamental limitation, but there are other challenging requirements when considering operation at short wavelengths. The first is the high value of the electron energy (a few hundred $\mathrm{MeV}$ or more) which implies that an electron storage ring must be employed. The second challenge is the problem of a reduced lifetime of the stored electron beam owing to the considerable energy loss after interaction with the field in the optical cavity. It should be noted that this situation is different from the one arising in undulators used in synchrotron radiation sources in order to reduce the width of a synchrotron radiation spectrum. (Undulators in synchrotrons are operated without an optical cavity so much less energy is extracted from the electrons.) Another challenging issue is the long undulator length, typically $25 \mathrm{~m}$, owing to the large number of periods needed to compensate the reduction of the intrinsic FEL gain with decreasing wavelength. The gain reduction is due to the fact that fewer electrons are available in each well of the ponderomotive potential.

A FEL first operated in the visible, at a wavelength of $650 \mathrm{~nm}$, in 1983 using the ACO storage ring at Orsay. This ring has been replaced by Super-ACO, which (like $\mathrm{ACO}$ ) primarily serves as a source of synchrotron radiation and only a fraction of the beam time is available for experiments on FEL operation in the UV. The shortest wavelength to date $(240 \mathrm{~nm})$ was achieved at Novosibirsk in 1989 but this project has been terminated for lack of funds.

A beam energy of at least $1 \mathrm{GeV}$ will be needed for operation in the vacuum ultraviolet and soft $\mathrm{x}$-ray regimes. Research is still in its infancy, with dedicated storage-rings under construction at Dortmund University and at Duke University in the USA (both projects aim at lasing down to $100 \mathrm{~nm}$, and possibly even to $10 \mathrm{~nm}$ ). For extension into the soft $x$-ray range it will be necessary to consider using larger storage rings, and a proposal along these lines has been made by the ESRF in Grenoble.

Many new experiments are brought into the realm of reality once soft $x$-ray laser radiation becomes available. It would be particularly exciting to operate in the "water window" between 2.4 and $4.4 \mathrm{~nm}$ (i.e., in 
between the $\mathrm{K}$-edges of carbon and oxygen) as this permits the study of living matter in its natural environment, the holy grail of many biologists. A demanding application is connected with the heavy-ion fusion scheme proposed by C. Rubbia [3] in which 3 $\mathrm{MW}$ of (peak) power, at a wavelength of 84 $\mathrm{nm}$, will be needed for photo-ionizing $\mathrm{Bi}^{+}$ ions.

\section{Microwave FELS}

There are also important applications for the FEL in the microwave region at the other end of the electromagnetic spectrum. The first is in high-energy physics, where the FEL is a candidate for a radiofrequency source for the next generation of colliders. This application is related to the need for much higher frequencies (needed to keep the accelerator dimensions within reasonable limits) than those which can be obtained using klystrons. The second application is in thermonuclear fusion. In future reactors such as the international fusion test reactor ITER now being designed, high-power microwaves will be absorbed locally to tailor the current-density profile. Even for the least demanding applications, several MW of power at frequencies ranging up to $300 \mathrm{GHz}$ will be needed. These requirements might be difficult to meet using the radiofrequency sources used at present, the so-called gyrotrons. Furthermore, gyrotrons are essentially non-tunable whereas rapid tunability will be of vital importance for suppressing plasma disruptions involving the essentially instantaneous deposition of the entire plasma energy onto the walls of the reactor that are usually initiated by a local instability. Rapid adjustment of the microwave frequency can be used to locally manipulate the plasma, with the aim of suppressing a growing instability before it evolves into an overall disruption. An induction linacdriven FEL carried out a short series of pilotscale plasma heating experiments at the MTX tokamak at the Lawrence Livermore National Laboratory in the USA before the work using this approach was discontinued.

Research groups around the world have operated FELs in the microwave range in the past. The required beam energy is typically one to several $\mathrm{MeV}$; the usual approach is to work at a beam current which is high enough to achieve saturation of the laser field in a single pass through the undu- lator so that there is no need for an optical cavity. Consequently, a compact accelerator with a pulse duration of typically $100 \mathrm{~ns}$ (such as a Marx generator) can be used. However, this duration is too short for the applications mentioned above, particularly those in nuclear fusion, where a continuous laser output is required. A free electron maser designed to produce $1 \mathrm{MW}$ of output power at $200 \mathrm{GHz}$ has been under development at the FOM-Institute for Plasma Physics since 1991. Operation in the continuous mode and at high efficiency will be possible via recovery of the beam charge and energy downstream of the undulator (a similar scheme is used in the low-power, far-infrared FEL at Santa Barbara).

As regards applications in high-energy physics, a programme to develop microwave FELs started recently at SLAC in Stanford, USA, where one of the investigators is R.M. Phillips who invented the ubitron some 25 years ago.

[1] Phillips R.M., Nucl. Instr. Meth. A 272 (1988) 1.

[2] Dlott D.D. \& Fayer M.D., IEEE J. Quant. Elect. 27 (1991) 2697.

[3] Europhysics News 23 (1992) 5.

\section{The THEMIS Telescope}

THEMIS is scheduled to be operational within three years. Its design will make it one of the most powerful instruments for the study of solar magnetism.

THEMIS is an acronym standing for Télescope Héliographique pour l'Etude du Magnétisme et des Instabilités Solaires. It started nearly 10 years ago as a uniquely French project, but approximately two years ago the Italian solar physics community joined the project with the signing of a formal agreement between the French Centre $\mathrm{Na}$ tional de la Recherche Scientifique (CNRS) and the Italian Consiglio Nazionale delle Ricerche (CNR). The CNR is participating through a financial contribution, construction of a technologically sophisticated dome, and the implementation of post-focus instrumentation with a filter of high spectral resolution.

With a very high polarimetric accuracy, an excellent spatial resolution, and the possibility of observing simultaneously the Stokes parameter profiles of many spectral lines, it is expected that THEMIS will provide a breakthrough in the study of solar magnetism at very high spatial resolutions $(<0.3$ arcsec). Accurate spectro-polarimetric observations will also be extremely important in studying the following related topics:

- energy transport mechanisms and velocity fields in magnetic structures;

- magneto-hydrodynamic turbulence and magneto-convection;

- the onset of instabilities, flares, and electric currents;

- fundamental processes in atomic physics (radiative transfer for polarized radiation, Hanle effect, impact polarization, etc.).

THEMIS is provided with an evacuated Cassegrain telescope with an azimuthal mount, and a Ritchey-Chrétien primary mirror of $90 \mathrm{~cm}$ in diameter. As the basic goal is the measurement of vector magnetic fields through the signatures observed in polarized radiation, normal-incidence optics is obviously necessary before the polarimetric analysis. Many modes of polarization analysis will be

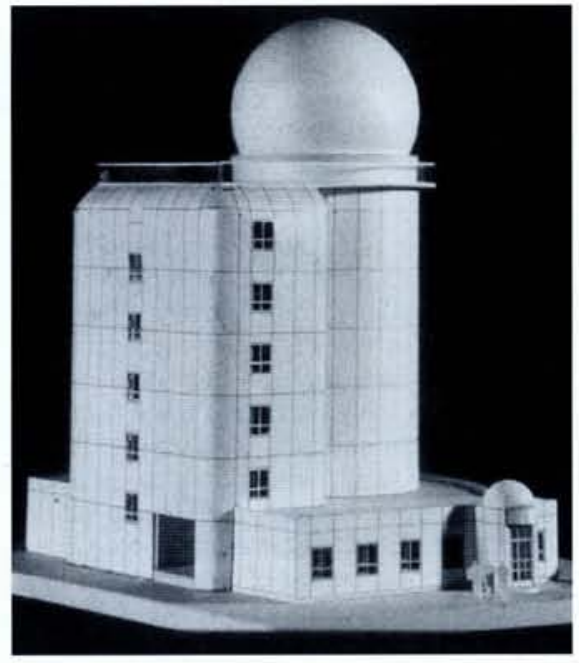

A model of THEMIS.

possible by exchanging automatically the analyzer and the related optics.

The study of solar magnetism implies high spatial resolution. This is made possible by the selection of an appropriate site (Izaña, Tenerife), and by correction of image motion by means of a tilting mirror, activated using a granulation tracker at frequencies exceeding $100 \mathrm{~Hz}$. The telescope will also be equipped for highly flexible spectroscopy to make possible the simultaneous observation of two Stokes parameters profiles in typically 10 spectral ranges, arbitrarily distributed along the spectrum (a long predisperser - with three exchangeable gratings - and an echelle-spectrograph will provide this unique possibility). Additive or subtractive modes will also be possible. Spectra will be recorded using several two-dimensional CCD cameras ( $288 \times 384$ pixels). Typically, one will be able to observe 10 spectral ranges in 2 polarization directions, at 384 wavelengths in 288 points along the slit (which implies $2.2 \times 10^{6}$ data readings!) every few seconds.

Several observing modes will be catered for. Besides the multi-line spectroscopy mode (probably the most important), it will also be possible to rapidly perform twodimensional spectroscopy in the so-called MSDP (Multichannel Subtractive Double Pass) mode. Alternatively, the telescope is operated in the so-called magnetograph mode using a slit in subtractive spectroscopy. The longitudinal component of the magnetic field and the Doppler velocity can thus be deduced. This observing mode is very fast and will be a great advantage in coordinated observations between ground- and spaceprobes (e.g., the SOHO satellite mission). Finally, the special Italian-designed filter that combines a tunable, universal birefringent filter with a tunable Fabry-Perot interferometer will allow two-dimensional spectroscopy.

Egidio Landi Degl'Innocenti

Dipartimento di Astronomia e

Scienza dello Spazio, Università di Firenze

\section{ECAMP 5}

The 5th European Conference on Atomic and Molecular Physics (ECAMP 5) will be held in Edinburgh, UK, on 3-7 April 1995. The Local Organizing Committee (Chair.: J.P. Connerade) and the International Programme Committee (Co-chairs: J.P. Connerade and $\mathrm{H}$. Hotop) have been appointed, and on behalf of the Atomic and Molecular Physics Divsion, AMPD members are invited to make written suggestions for titles and speakers before 31 January 1994. Most of the programme will be decided in April 1994, and the first circular mailed in May 1994. Correspondence should be sent to Dr. N.J. Mason, Secretary, ECAMP 5, Dept. of Physics \& Astronomy, University College, Gower St., London WC1E 6BT, UK (tel./fax: +44-71-380 77 97 / 38071 45; email: ucaps7n @ ucl.ac.uk). 